\title{
Sticky Rules: Integration Between Abstract Rules and Specific Actions
}

\author{
Ulrich Mayr and Richard L. Bryck \\ University of Oregon
}

\begin{abstract}
The authors manipulated repetitions and/or changes of abstract response rules and the specific stimulusresponse $(\mathrm{S}-\mathrm{R})$ associations used under these rules. Experiments 1 and 2, assessing trial-to-trial priming effects, showed that repetition of complete $\mathrm{S}-\mathrm{R}$ couplings produced only benefits when the rule also repeated (i.e., rule-S-R conjunctions) but costs when identical $\mathrm{S}-\mathrm{R}$ couplings repeated while rules changed. In Experiments 3 and 4, the authors manipulated amount of experience with specific rule-S-R conjunctions and demonstrated integration between rules and $\mathrm{S}-\mathrm{R}$ couplings in terms of cumulative practice effects. However, unlike short-term priming effects, cumulative practice supported generalization of experience with specific S-R couplings across rule boundaries (Experiment 4). Results are discussed in terms of constraints on models of hierarchical control and in terms of qualitatively different ways in which people profit from very recent experiences (i.e., all-or-none access to working memory representations) versus cumulative experience (i.e., similarity-based retrieval from long-term memory).
\end{abstract}

In models of cognitive control, it is often posited that selection occurs in a hierarchical manner (e.g., Koechlin, Ody, \& Kounelher, 2003; Norman \& Shallice, 1986). On one level, an abstract set of rules, stimulus dimensions, or possible response options is chosen, which in turn constrains selection on a lower level that then determines the specific response. Behavioral and brain imaging evidence (e.g., Gopher, Armony, \& Greenshpan, 2000; Koechlin et al., 2003) is generally compatible with such a distinction between levels of selection. However, an open question is how, exactly, the different levels of selection are interrelated.

One theoretical possibility is that control is strictly hierarchically organized: Once an instructed task set has configured the cognitive system, its job is done, and it plays no further role in the lower level selection process itself. For example, if a task requires a left keypress for red stimuli and a right keypress for green stimuli, then the task-level codes would be responsible for configuring the cognitive system to process only the color of the object (thereby ignoring other dimensions) and to establish the relevant S-R links. However, according to this account, the task-level codes are irrelevant during the response-selection process itself and therefore would not make contact with the stimulus or response codes involved in an actual selection episode. The advantage of this type of modular selection architecture is that changes could be carried out on one level without affecting efficiency on the remaining levels. For example, if a particular action is learned under Rule A and then reused under Rule B, full transfer regarding the lower level action selection process should be expected.

A second possibility is that codes representing abstract rules and codes representing concrete actions become integrated into a common representation during selection. We refer to this as the crosslevel integration model. According to this model, the codes responsible for task-specific selection settings are active during the

Ulrich Mayr and Richard L. Bryck, Department of Psychology, University of Oregon.

Correspondence concerning this article should be addressed to Ulrich Mayr, Department of Psychology, University of Oregon, Eugene, OR 97403. E-mail: mayr@ darkwing.uoregon.edu response selection process and can become associated with lower level stimulus-response codes. A straightforward implication of this model is that even when two selection episodes are based on identical lower level stimulus or response codes, they would be integrated into distinct representations if they occur within different abstract rule settings. In contrast with the strictly hierarchical model, no transfer across abstract rules would be possible. Although an integrative process that builds associations across levels seems to contradict the very definition of an abstract rule, it may nevertheless be useful. For example, with such integrated representations in place, activation of an abstract rule may coactivate a specific action that is frequently associated with that particular rule (or vice versa) and thereby better prepare the cognitive system for regularities that are not contained within one particular level of selection.

In this research, we explored to what degree integration across levels of selection occurs. We developed a new variant of the so-called task-switching paradigm, which allows an unambiguous test of the degree of cross-level integration. Using this paradigm, in Experiments 1 and 2, we examined integration across levels in the context of trial-to-trial priming effects. In Experiments 3 and 4, we adapted the same basic paradigm to look at potential crosslevel integration effects of cumulative experience.

\section{Levels of Selection in the Task-Switching Paradigm}

A paradigm that allows explicit consideration of both higher level selection of task rules and lower level selection of stimuli or responses is the so-called task-switching paradigm (e.g., Mayr, 2002; Meiran, 1996; Rogers \& Monsell, 1995). As in standard choice response time (RT) paradigms, participants execute selections between simple responses. However, in addition, they need to select between rules governing the lower level response-selection process, often called task sets. Changes of task sets usually produce substantial switch costs, and much of task-switching research has focused on identifying sources of these costs (e.g., Allport, Styles, \& Hsieh, 1994; Mayr \& Keele, 2000; Mayr \& Kliegl, 2000, 2003; Meiran, 1996; Rogers \& Monsell, 1995). One important result from this research is that switch costs and manipulations 
affecting the difficulty of lower level selection often behave in a strikingly additive manner (e.g., Allport et al., 1994; Rubinstein, Meyer, \& Evans, 2001; for a review, see Gopher et al., 2000; for a theoretically important exception, see Mayr \& Kliegl, 2000). Such additivity has been taken as evidence in favor of independence between levels of selection. ${ }^{1}$

There is, however, one empirical phenomenon that is suggestive of integration between levels of selection. When task sets repeat across trials, typical response-repetition benefits are observed (e.g., Rogers \& Monsell, 1995). In contrast, when the task sets change across trials, response-repetition effects are usually eliminated and often even reversed. This Switch $\times$ Repetition interaction has been interpreted in terms of a strong integration between task-set codes on the one hand and simple-action codes on the other. For example, according to Schuch and Koch (2004), executing a response in the context of a particular task set associates this response with the task-specific meaning (see also Meiran, 2000). Response-repetition costs in case of a switch arise either because alternative meanings are suppressed, and therefore are less available after a switch (Schuch \& Koch, 2004), or because an extra unbinding process is necessary before an alternative meaning can be associated with the response code (Hommel, 2004).

Although the Switch $\times$ Response Repetition interaction seems highly suggestive of cross-level integration, it could also arise simply on the level of stimulus-response codes without requiring assumptions about across-level integration. In typical taskswitching experiments, different task sets require participants to focus on different stimulus aspects (e.g., color vs. shape). Thus, in case of a task-set repetition, response repetitions usually also imply a repetition of the stimulus attribute (e.g., red $\rightarrow$ right key followed by red $\rightarrow$ right key). In contrast, when the task set changes, response repetitions occur while at the same time, the attended stimulus attribute changes (e.g., red $\rightarrow$ right key followed by square $\rightarrow$ right key). Even if the exact same stimulus is presented on two consecutive trials (e.g., a red square when both red and square require a right-key response), the attended stimulus attribute has shifted (e.g., from red to square). Thus, if specific stimulusresponse couplings are in fact the basis of repetition benefits, such benefits might disappear simply because of the change in attended stimulus attribute that usually goes along with a task switch.

We can consult response-repetition research to demonstrate the significance of this argument. A common strategy in this domain is to pair two or more stimuli with each response. This allows examining effects of response repetitions that are not accompanied by stimulus repetitions. For example, in Marczinski, Milliken, and Nelson's study (2003), participants responded to four different colors using a 4:2 stimulus-response mapping. When the color changed but the response repeated, a substantial repetition cost was observed, compared with when both stimulus and response changed (for similar results, see Campbell \& Proctor, 1993; Pashler \& Baylis, 1991; Quinlan, 1999). Note that in this situation, no active task set is required, because stimuli uniquely determine responses. In other words, a Switch $\times$ Response Repetition interaction can arise as a purely low-level phenomenon that requires no assumptions about codes on the task-set level.

These observations suggest that repetition-priming effects in the context of standard task-switching situations do not provide a good diagnostic for cross-level integration. However, this does not mean that such cross-level integration does not occur. An unambiguous empirical test of cross-level integration would require a situation in which the exact same stimulus (including the way it is interpreted) and the exact same response can repeat across a task-switch transition. If the standard Switch $\times$ Response Repetition interaction is caused exclusively by changes in the attended stimulus aspect, then repetition benefits should be observed across such a switch in task without changes in stimulus aspects. However, if there is true cross-level integration, then repetition costs should be observed in this situation, even though stimulus and response aspects are constant.

\section{Independent Variation of Abstract Rules and Specific Actions}

In Experiments 1 and 2 described here, we attempted to construct such a situation, in which higher level rules can change without requiring a change in the lower level stimulus-response couplings. Our paradigm was adapted from the spatial-rule task introduced by Mayr (2002; see Figure 1). On each trial, participants were presented with a square with a circle in one of its corners. Four response keys were arranged in a manner that was spatially congruent with the four corners of the square. Participants determined responses by the conjunction between the circle's location and one of four spatial response rules (vertical, horizontal, clockwise or counterclockwise). For example, if the circle location was upper right and the rule was vertical, the correct response was to the key corresponding to the lower right corner (i.e., reflecting a move of the stimulus location along the vertical dimension).

The critical feature of this paradigm is that four of the six possible rule pairings shared identical stimulus-response couplings. For example, both for the horizontal and the clockwise rule, the upper left and the lower right stimulus positions led to identical response positions, whereas the upper right and the lower left stimulus positions led to different response positions. This allowed us to create trial-to-trial transitions in which stimuli and responses repeated, but the rule governing this stimulus-response coupling changed. In addition, all other possible transitions could occur between consecutive trials with rule changes: stimulus-only repetitions, response-only repetitions and repetitions of neither stimulus nor response. In case of rule repetitions, there were only two possible constellations: Either both stimulus and response repeat, or both change.

An additional variable we manipulated was the cue-stimulus interval (CSI), that is, the interval between the cue indicating the upcoming task rule and the stimulus. We included this variable to examine the possibility that integration between rules and lower level stimulus-response aspects is a function of temporal proximity between rule selection and stimulus-response selection.

\footnotetext{
${ }^{1}$ As mentioned by one reviewer, the assertion of additivity between set-level and response-level effects may be somewhat overstated for one particular phenomenon, the Switch $\times$ Response Congruency interaction for the relevant versus irrelevant task. In some cases, overadditivity for this factor has been reported (e.g., Meiran, 1996). However, in this context, the Switch $\times$ Congruency interaction is a somewhat odd phenomenon because response congruency can be affected by set-level phenomena for relatively trivial reasons. To be specific, when participants occasionally forget to switch, this will likely show up as fast RTs on congruent trials (because no switch has taken place) but as errors on incongruent trials (which are not analyzed). The result will be larger congruency effects on switch than on no-switch trials.
} 

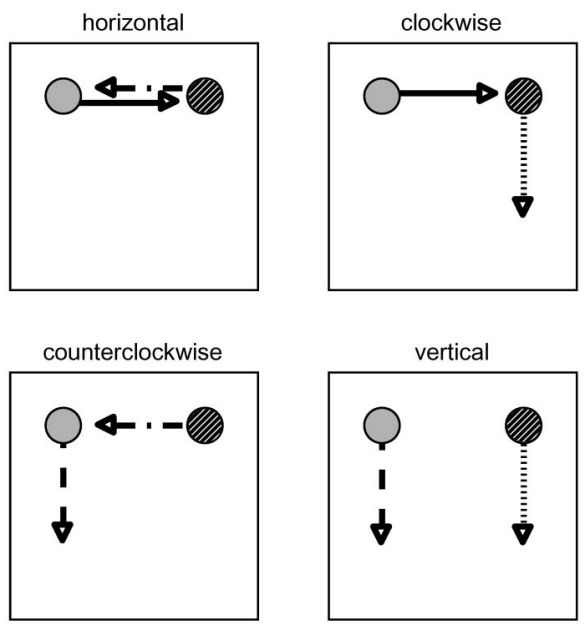

Figure 1. Sample constellations of stimuli, responses, and spatial rules. Participants responded with one of four response keys arranged in terms of a square. Responses (indicated by the arrows) were codetermined by the location of the circle in the frame and the spatial translation rule. Arrows with identical line patterns indicate stimulus-response couplings that are identical across a pair of rules. For example, the response to a circle in the upper left corner is to the key in the upper right corner for both the horizontal and the clockwise rule. The actual stimulus presented to participants contained only one circle per frame, and no arrows were shown.

\section{Experiment 1}

\section{Method}

Participants. In exchange for course credit or \$7, 18 University of Oregon students participated in a single-session experiment.

Task and stimuli. Stimulus presentation occurred on a 17-in. Macintosh monitor. The stimulus display contained a frame in the form of a square with side lengths of $8 \mathrm{~cm}\left(9.1^{\circ}\right)$. On each trial, a circle with a diameter of $1 \mathrm{~cm}$ appeared in one of the four corners of the frame (see Figure 1). Participants entered responses using four keys on the standard Macintosh keyboard with the same spatial arrangement as the four circle locations $(1,2,4$, and 5) on the numerical keypad. Participants were instructed to rest the index finger of their preferred hand in the middle among the four keys and to move the finger to the correct key. After pressing the key, participants were instructed to bring back the index finger to the center position. Correct keys were cospecified by the circle location and the currently relevant rule. For the horizontal rule, a horizontal shift of the circle position led to the response location; for the clockwise rule, the clockwise translation of the circle position led to the response location. The vertical and the counterclockwise rules were to be used in an analogous manner. The relevant rule for each trial was presented through verbal abbreviations (ver, hor, clock, and counter) immediately above the stimulus frame and was visible until a response was entered. In case of an incorrect response, the word Error was presented on the screen for $500 \mathrm{~ms}$.

Relevant action rules and circle positions were selected randomly for each trial. The time between a response and the next stimulus (RSI) was $1,000 \mathrm{~ms}$. The verbal cue indicating the next relevant rule was presented within this interval either $900 \mathrm{~ms}$ or $100 \mathrm{~ms}$ before presentation of the stimulus.

Procedure. Participants completed 12 blocks of 96 trials each. For half of the participants, CSI was short in the first half of blocks and long in the second half of blocks; for the other half of participants, the reverse sequence was used. The first block of each half (Blocks 1 and 7) was regarded as practice.

\section{Results}

Given the random selection of all selection-relevant parameters for each trial, transitions between all four possible rules could occur. However, transitions between the horizontal and the vertical rule as well as transitions between the clockwise and the counterclockwise rule never involved repetitions of complete S-R couplings. In order to not bias the critical analyses through inclusion of these transitions, we eliminated them from all analyses. Also, error trials and trials after an error, as well as all RTs larger than $4,000 \mathrm{~ms}$, were eliminated (i.e., $0.6 \%$ of all trials were eliminated) Unless noted otherwise, all statistical results reported were reliable on the $p=.05$ level.

Figure $2 \mathrm{~A}$ and Table 1 show the critical data pattern. As is evident, substantial repetition benefits were obtained when the rule, the stimulus, and thus also the response, repeated. However, when the complete stimulus-response coupling but not the rule repeated, a cost was observed. This result is consistent with the assumption of cross-level integration between set-level and stimulus-response-level codes.

We analyzed this data pattern in two steps. First, we compared the rule-repetition and rule-change conditions for those S-R constellations that were constant across both conditions, namely the complete $\mathrm{S}-\mathrm{R}$ repetitions and the complete $\mathrm{S}-\mathrm{R}$ changes. An analysis of variance (ANOVA) with the factors CSI, rule repetition, and $\mathrm{S}-\mathrm{R}$ repetition revealed a highly reliable Rule Repetition $\times \mathrm{S}-\mathrm{R}$ Repetition interaction, $F(1,17)=61.6, M S E=$ 5,328.1. In addition, the main effects for CSI, $F(1,17)=5.2$, $M S E=18,787.5$; rule repetition, $F(1,17)=69.0, M S E=$ $13,358.2$; and response repetition, $F(1,17)=35.8, M S E=$ $3,676.5$, were reliable. The critical Rule Change $\times$ S-R Repetition interaction could be attributed both to a reliable responserepetition benefit in case of rule repetitions, $F(1,17)=84.7$, $M S E=5,168.1$, and a reliable response-repetition cost in case of rule changes, $F(1,17)=6.0, M S E=12,595.5$. There was no reliable interaction associated with the CSI factor, all $F \mathrm{~s}(1,17)<$ $1.4, p>.25$. Error results generally mirrored RT results, including a highly reliable Rule Change $\times$ Response Repetition interaction, $F(1,17)=14.4, M S E=400.3$.

In a second step, we focused on the rule-change condition in order to assess potential independent or combined effects of stimulus or response repetitions/changes. We conducted an ANOVA with the factors CSI, stimulus repetition, and response repetition. The only relevant reliable effect was associated with the response repetition factor, $F(1,17)=9.3, M S E=2,030.6$, suggesting a general cost in case of response repetitions. However, numerically, response repetitions were associated with higher costs in the case of stimulus repetitions $(M=42 \mathrm{~ms})$ than they were in the case of stimulus changes $(M=3 \mathrm{~ms})$, and the relevant interaction only approached significance, $F(1,17)=2.52, M S E=5,595.8, p=$ .13. No relevant error effects were obtained here.

\section{Experiment 2}

The main result of Experiment 1 was that even identical stimulus-response repetitions produced repetition costs rather than benefits in case of a rule change. This is consistent with the notion that rule-level codes become integrated with low-level stimulus and response codes. However, there is one possible qualification. In Experiment 1, rules varied randomly on a trial-by-trial basis and 


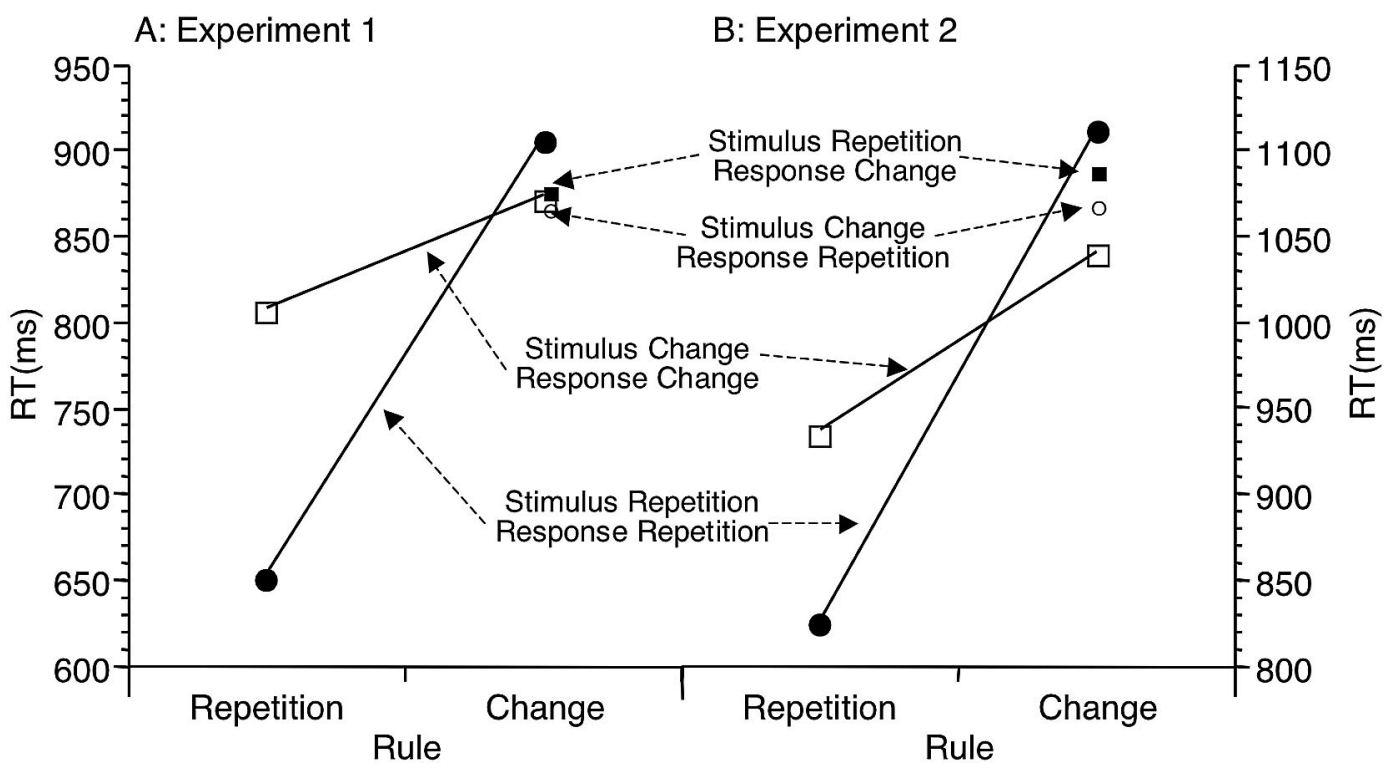

Figure 2. Mean response times (RTs) for Experiments 1 (A) and 2 (B) as a function of rule change-repetition, stimulus change-repetition, and response change-repetition. Note that stimulus changes-response repetitions and stimulus repetitions-response changes are structurally impossible for the rule-repetition condition.

therefore had to be signaled through a visual cue. Perhaps the critical aspect that determines repetition benefits or costs is not the change of rule per se, but the change of the stimulus that signals the rule. To be specific, the cue stimulus and the response-relevant stimulus may be integrated into a compound cue (e.g., see Logan $\&$ Bundesen, 2003), in which case, assumptions about rule-level codes are not necessary to explain the pattern of repetition effects observed in Experiment 1. Therefore, in Experiment 2, we implemented the spatial-rule task in form of the so-called alternate-runs paradigm. Participants alternated between two tasks every two trials. Progression through the alternation sequence was indicated by a spatial cue with equivalent perceptual changes for rule repetitions and rule changes. Thus, if a similar lack of repetition benefits for complete stimulus-response repetitions were observed here, it could not be explained in terms of a change in cue alone.

\section{Method}

Participants. In exchange for course credit or $\$ 7,14$ University of Oregon students participated in a single-session experiment.

Table 1

Mean Response Times (RTs) in Milliseconds and Mean Error Percentages for All Design Conditions of Experiment 1

\begin{tabular}{|c|c|c|c|c|c|c|}
\hline \multirow[b]{2}{*}{ Rule } & \multirow[b]{2}{*}{ Stimulus } & \multirow[b]{2}{*}{ Response } & \multicolumn{2}{|c|}{ Mean RT } & \multicolumn{2}{|c|}{ Mean error } \\
\hline & & & $M$ & $S D$ & $M$ & $S D$ \\
\hline \multicolumn{7}{|c|}{$\mathrm{CSI}=100$} \\
\hline Repetition & Repetition & Repetition & 671 & 151 & .5 & 1.1 \\
\hline Repetition & Change & Change & 832 & 136 & 3.5 & 2.7 \\
\hline Change & Repetition & Repetition & 943 & 186 & 3.5 & 3.4 \\
\hline Change & Repetition & Change & 892 & 192 & 2.5 & 3.7 \\
\hline Change & Change & Repetition & 887 & 202 & 4.2 & 3.9 \\
\hline Change & Change & Change & 906 & 103 & 3.9 & 5.0 \\
\hline \multicolumn{7}{|c|}{$\mathrm{CSI}=900$} \\
\hline Repetition & Repetition & Repetition & 638 & 140 & .3 & 1.0 \\
\hline Repetition & Change & Change & 787 & 178 & 2.0 & 2.3 \\
\hline Change & Repetition & Repetition & 876 & 235 & 4.2 & 6.0 \\
\hline Change & Repetition & Change & 843 & 201 & 3.3 & 4.1 \\
\hline Change & Change & Repetition & 868 & 218 & 3.6 & 4.0 \\
\hline Change & Change & Change & 843 & 214 & 3.4 & 3.4 \\
\hline
\end{tabular}

Note. Entries shown in italics were relevant for the primary analysis of complete stimulus-response repetition effects as a function of rule change/repetition. CSI $=$ Cue-Stimulus interval. 
Task and stimuli. We used the same tasks and stimuli that we used in Experiment 1. Instead of an explicit task cue, the sequence of trials was cued by a green star that moved clockwise around the corners of the stimulus frame in a trial-by-trial manner. Depending on the specific block of trials, a star at the top two locations signaled either the horizontal or the vertical task, whereas a star on the bottom two locations signaled either the clockwise or the counterclockwise task. Thus, horizontal shifts of the star were always associated with rule repetitions; vertical shifts were always associated with rule changes. In either case, a marked perceptual change occurred between trials. In case of an incorrect response, the word Error and the relevant rules were presented next to each corner of the square (e.g., the word vertical next to the upper two corners and the word clockwise next to the bottom two corners). This display stayed on until the correct response was entered. This procedure allowed participants to reorient themselves within the sequence of events in case of an error.

For each block of trials, only two rules were relevant (one from the horizontal-vertical pair and the other from the clockwisecounterclockwise pair). The alternate-runs-of-two schema determined the sequence of rules, whereas stimulus and response aspects were determined randomly. The RSI was either $100 \mathrm{~ms}$ or $1,000 \mathrm{~ms}$.

Procedure. At the beginning of the session, participants were familiarized with the task in at least two 10-trial practice blocks, which could be repeated on demand. Throughout, participants were informed prior to each block which two rules would be relevant. In case of an error, the task sequence was presented, the current sequence position was highlighted, and participants could initiate the posterror trial at their leisure. Actual testing occurred in two sets of twenty 24-trial blocks. Within sets, the sequence of rule pairs for each block was determined randomly with the constraint that each of the four relevant rule pairs occurred in 6 blocks. For one of the two sets, RSI was $100 \mathrm{~ms}$; for the other, it was $1,000 \mathrm{~ms}$. RSI was counterbalanced across participants.

\section{Results}

We eliminated error trials and trials after an error within the preceding trial, as well as all RTs larger than 4,000 ms (i.e., we eliminated $0.7 \%$ of all trials).
Figure $2 \mathrm{~B}$ shows the critical data pattern, and Table 2 contains RTs and error scores from all design cells. Again, the effect of stimulus-response repetitions was conditional on whether the rule changed or repeated with benefits in case of rule repetitions and costs in case of rule changes. This pattern constitutes a clear replication of the results obtained in Experiment 1.

As in Experiment 1, an ANOVA with the factors RSI, rule repetition, and $\mathrm{S}-\mathrm{R}$ repetition revealed a highly reliable Rule Repetition $\times$ S-R Repetition interaction, $F(1,13)=44.0, M S E=$ 5,272.1. Also, the main effects for CSI, $F(1,13)=17.5, M S E=$ 177,509.0; and rule repetition, $F(1,13)=67.6, M S E=15,872.9$ were reliable. Again, the critical Rule Change $\times$ S-R Repetition interaction was due both to a reliable response-repetition benefit in case of rule repetitions, $F(1,13)=18.5, M S E=9,131.7$, and to a reliable response-repetition cost in case of rule changes, $F(1$, $13)=12.7, M S E=5,741.5$. Unlike in Experiment 1, however, general rule-repetition effects became smaller with increasing RSI, $F(1,13)=16.4, M S E=102,184.7$, and there was an interaction involving all three factors, $F(1,13)=11.2, M S E=2,058.2$. As inspection of Table 2 reveals this interaction was due to the fact that the cost associated with $\mathrm{S}-\mathrm{R}$ repetitions was much larger for the short RSI (106 ms) than it was for the long RSI (39 ms).

Error results did not correspond with RT results in this experiment. There were main effects for rule repetition, $F(1,13)=8.4$, $M S E=304.6$, and for response repetition, $F(1,13)=7.9, M S E=$ 913.0; however, the interaction was not reliable, $F(1,13)=0.4$, $M S E=660.6$. In fact, fairly similar repetition benefits were observed both for rule repetitions $(M=1.7 \%)$ and rule changes $(M=1.5 \%)$.

At first sight, the error pattern may seem inconsistent with the conclusion suggested by our results this far, namely that positive priming effects do not cross the border of different rules. However, there is an unavoidable, structural confound in the paradigm we used that may have produced the error response-repetition benefit

Table 2

Mean Response Times (RTs) in Milliseconds and Mean Error Percentages for All Design Conditions of Experiment 2

\begin{tabular}{|c|c|c|c|c|c|c|}
\hline \multirow[b]{2}{*}{ Rule } & \multirow[b]{2}{*}{ Stimulus } & \multirow[b]{2}{*}{ Response } & \multicolumn{2}{|c|}{ Mean RT } & \multicolumn{2}{|c|}{ Mean error } \\
\hline & & & $M$ & $S D$ & $M$ & $S D$ \\
\hline \multicolumn{7}{|c|}{$\mathrm{RSI}=100$} \\
\hline Repetition & Repetition & Repetition & 952 & 267 & 2.4 & 2.6 \\
\hline Repetition & Change & Change & 1,086 & 361 & 4.0 & 3.6 \\
\hline Change & Repetition & Repetition & 1,328 & 307 & 3.6 & 4.0 \\
\hline Change & Repetition & Change & 1,259 & 326 & 4.1 & 6.2 \\
\hline Change & Change & Repetition & 1,253 & 426 & 7.0 & 3.8 \\
\hline Change & Change & Change & 1,222 & 377 & 4.9 & 2.5 \\
\hline \multicolumn{7}{|c|}{$\mathrm{RSI}=1,000$} \\
\hline Repetition & Repetition & Repetition & 703 & 150 & 1.6 & 2.3 \\
\hline Repetition & Change & Change & 789 & 169 & 3.4 & 2.5 \\
\hline Change & Repetition & Repetition & 901 & 216 & 2.6 & 2.9 \\
\hline Change & Repetition & Change & 918 & 207 & 6.6 & 5.3 \\
\hline Change & Change & Repetition & 884 & 190 & 5.3 & 4.9 \\
\hline Change & Change & Change & 862 & 182 & 4.2 & 2.6 \\
\hline
\end{tabular}

Note. Entries shown in italics were relevant for the primary analysis of complete stimulus-response repetition effects as a function of rule change/repetition. RSI $=$ time between a response and the next stimulus. 
in case of rule changes in this experiment. To be specific, by definition, each of those S-R couplings that are identical for two different rules are also congruent, so that no matter which of the two rules is relevant, the response to this particular location will be identical. Typically, in task-switching research, response congruency is an important factor that affects both RTs and errors (e.g., Meiran, 1996; Rogers \& Monsell, 1995). Moreover, whereas all constellations with identical S-R couplings across rules are congruent, constellations for which both the stimulus and the response change are a combination of both congruent and incongruent trials. It is likely that the congruency factor played only a minor role in Experiment 1, because all four possible rules were used, and a particular constellation was congruent with regard to one relevant alternate rule but incongruent with regard to the other. However, in Experiment 2, for a given block, only two rules were relevant, so that particular S-R couplings would be either consistently congruent or incongruent that block. In those conditions in which the congruency effect could be assessed (because they contain both congruent and incongruent trials), it was reliable: in the rule-repeat condition $(M=1.4 \%), t(13)=2.7$, and, more critically, also in the rule-change condition with complete $\mathrm{S}-\mathrm{R}$ changes $(M=3.6 \%)$, $t(13)=4.3$ (no comparable results were obtained for RTs). Further, the response-repetition error benefit obtained in case of response changes turned into small and nonsignificant cost $(M=$ $0.15 \%$ ) after we eliminated all incongruent trials from the constellation with rule changes and complete $\mathrm{S}-\mathrm{R}$ changes (and thus, eliminating the congruency confound otherwise present in this comparison). Eliminating incongruent trials did not alter RT results. In sum, we can be fairly confident that after considering the congruency confound, both the RT and the error pattern are consistent with the view that true repetition priming benefits the area constrained by rule boundaries.

In light of the foregoing discussion of the congruency confound, the second set of analyses focusing on rule-change trials should be considered with caution, because here the confound cannot be eliminated (i.e., all constellations in which only the stimulus or the response changes are incongruent). An ANOVA with the factors CSI, stimulus repetition, and response repetition revealed a reliable effect associated with the stimulus repetition factor, $F(1,13)=$ 6.6, $M S E=9,096.5$, suggesting a general cost in case of stimulus repetitions and a tendency for a response repetition cost, $F(1$, 13) $=3.99, M S E=4,732.6$. There was no trace of a Stimulus Repetition $\times$ Response Repetition interaction, $F(1,13)=0$. No reliable error results were obtained here.

\section{Discussion of Experiments 1 and 2}

The central result of Experiments 1 and 2 was that repetitionpriming benefits were constrained to the combined repetition of task sets, stimuli, and responses. What is most striking is that an exact repetition of $\mathrm{S}-\mathrm{R}$ couplings under the condition of changing rules led to a repetition cost. In fact, the repetition costs in the case of complete S-R couplings were greater, albeit not always statistically significant, than stimulus or response repetitions alone. This pattern of results is strongly suggestive of integration between rule-level and $\mathrm{S}-\mathrm{R}-\mathrm{level}$ codes.

A remaining issue is the role that task cues might play in the observed repetition effects. Logan and Bundesen (2003) have recently suggested that in paradigms with explicitly cued tasks, participants do not actually switch between abstract tasks or rules but, rather, use the cue to form a cue-stimulus compound, which then uniquely determines the response. We attempted to address this problem by using the alternate-runs paradigm in Experiment 2, in which identical changes in external cues occurred between no-switch and switch trials (i.e., the star wandering from position to position). However, it is possible that participants did not represent the cue signal in terms of its actual position but in terms of task-relevant categories (i.e., top $=$ Task 1 and bottom $=$ Task 2). In this case, position changes would not be equivalent for no-switch and switch transitions. To counter this argument, it would be useful to show that the characteristic cost-benefit priming pattern is obtained even when participants perform the alternate-runs sequence entirely endogenously, that is, without the help of external cues. In a separate experiment, we included such a condition (along with other conditions not relevant here). The pattern of cost and benefit effects was very similar to the one obtained in Experiment 2: a substantial repetition benefit in the case of a rule repetition and a repetition cost in the case of a rule switch that was nearly as large. Thus, on the basis of these new data, it seems that the pattern of priming effects arises from integration between abstract rules and stimulus-response codes and not from a compound stimulus that simply combines the external cue with the stimulus-response codes.

Another interesting issue regards the role of the cue-stimulus interval in Experiment 1 and of the response-stimulus interval in Experiment 2. The pattern of priming effects was basically unaffected by the cue-stimulus interval in Experiment 1, in which the cue-stimulus interval was manipulated but the response-stimulus interval was constant. This suggests that integration of rule-level and stimulus-response-level codes is not a matter of temporal proximity between selection processes on the two levels. At first sight, this seems inconsistent with the finding of a marked reduction of the cost-benefit pattern observed in Experiment 2 for the long response-stimulus interval. However, variation of the response-stimulus interval in an alternate-runs paradigm affects both the time to select the rule prior to the stimulus and the total time between trials (which was constant in Experiment 1). As a consequence, for the short response-stimulus interval in Experiment 2, there was less opportunity for time-based or interferencebased disintegration of representations carried over from the preceding trial than for the long response-stimulus interval (for a generally similar result, see Schuch \& Koch, 2004). Thus, the reduction of the cost-benefit pattern as a function of the responsestimulus interval may be an important hint toward the time-graded nature of representations that integrate set-level and stimulusresponse-level codes.

\section{Experiment 3}

Our results so far suggest that integrated representations between low-level and high-level codes form the basis of short-term priming effects. In Experiment 3, we asked whether longer term priming and learning effects are also constrained by rule-level representations. To be specific, we examined whether longer term effects specific to particular rule-stimulus-response conjunctions can be obtained within our paradigm. An additional important question was to what degree learning of such conjunctions is a matter of explicit-declarative knowledge, or whether it can occur implicitly. 
We adapted the basic trial-by-trial cuing paradigm we used in Experiment 1. However, unbeknown to participants, for each rule there were two stimulus locations (and therefore also responses) that occurred with higher frequency $(p=.40)$ than each of the other two locations $(p=.10)$. For each of the four rules, there was a different pair of high-frequency locations, so that across rules, each stimulus location, each response, and each stimulus-response coupling occurred equally often. In other words, if, and only if, participants were sensitive to rule-stimulus-response conjunctions, faster and/or more accurate performance for the high-frequency conjunctions should have been obtained.

\section{Method}

Participants. In exchange for course credit or $\$ 7,12$ University of Oregon students participated in a single-session experiment.

Task and stimuli. We used the same tasks, stimuli, and cues that we used in Experiment 1. Unlike those in Experiment 1, rules were selected randomly with the constraint of no immediate rule repetitions. We avoided such repetitions in order to reduce saliency of rule-specific high-frequency stimuli. Stimuli were selected conditional on the current rule. For each rule, two stimulus locations occurred with high probability (each $p=.40$ ), whereas the other two occurred with lower probability (each $p=.10$ ). The constellation of rule-specific frequent stimulus locations was counterbalanced across participants. The RCI was $800 \mathrm{~ms}$, and the CSI was $100 \mathrm{~ms}$.

A frequency-judgment task was presented at the end of the experiment to assess explicit awareness of contingencies. At this point, participants were informed that some rule-stimulus locations had occurred more frequently than others. Then, each of the 16 rule-stimulus locations was presented once in random order, and participants had to make an unspeeded, two-choice response to indicate whether the constellation had occurred with low or high frequency.

Procedure. Participants began with one 50-trial practice block and then completed nine 100-trial test blocks followed by the 16-trial frequency-judgment block (i.e., 1 trial for each unique rule-stimulus constellation).

\section{Results and Discussion}

Error trials and trials after an error, as well as all RTs larger than $4,000 \mathrm{~ms}$ (i.e., $1.0 \%$ of all trials) were excluded. To analyze frequency effects on performance, we aggregated across the first three, the second three, and the third three blocks. Figure 3 shows the critical data. High-frequency rule-stimulus constellations produced faster RTs than low-frequency rule-stimulus constellations. Statistically, the factor was highly reliable, $F(1,11)=12.52$, $M S E=4,012.53$, as was the linear contrast across the three practice segments, $F(1,11)=14.48, M S E=1,538.12$. The Frequency $\times$ Linear Practice Contrast interaction approached the reliability criterion, $F(1,11)=2.33, M S E=3,609.1, p=.16$. Error rate was slightly lower for high-frequency constellations than it was for low-frequency constellations $(M=2.5 \%, S D=2.3$ vs. $M=3.6, S D=3.5)$; however, this effect was not reliable, $F(1$, 11) $=2.64, M S E=853.3, p>.1$.

Explicit frequency judgments revealed some explicit knowledge about the relative frequencies of rule conjunctions. Participants identified $68.8 \%(S D=16 \%)$ of high-frequency conjunctions correctly. However, they also identified $52.8 \%(S D=16 \%)$ of low-frequency conjunctions as having occurred with high frequency. This difference was reliable, $t(11)=2.60, p<.05$.

In order to assess the degree to which awareness of highfrequency conjunctions may have been critical for RT learning

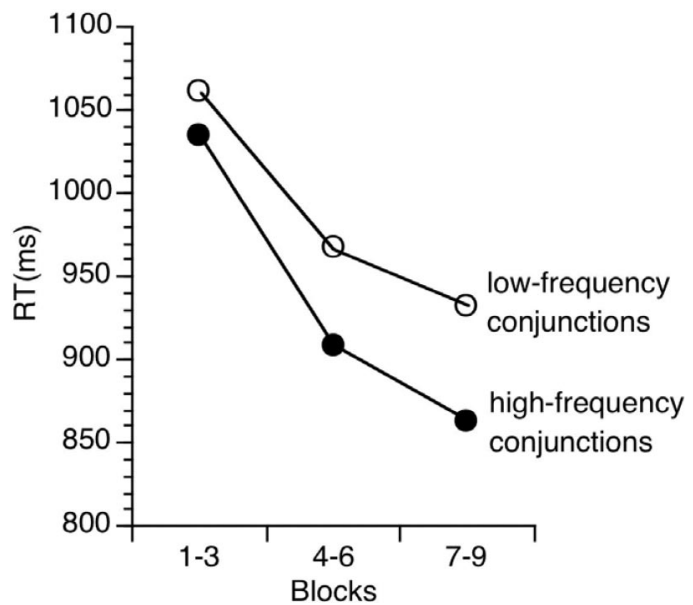

Figure 3. Response times (RTs) for Experiment 3 as a function of stimulus-response rule conjunction frequency and practice.

effects (or vice versa), we conducted a median split on the difference score for frequency judgments of high-frequency versus low-frequency conjunctions. Low-awareness participants had a difference score of $0 \%(S D=11.1)$, and high-awareness participants had a difference score of $33.3 \%$ ( $S D=17.1$ ). High versus low awareness as between-subjects factor did not reliably modulate the RT frequency effect (all $F \mathrm{~S}<1.2$ ). If anything, unaware participants showed larger priming effects $(69 \mathrm{~ms}, S D=65)$ than did aware participants $(37 \mathrm{~ms}, S D=32)$. Thus, even though awareness of conjunction frequencies developed in a subset of the participants, there is no evidence that it was critical for the RT benefits associated with high-frequency conjunctions.

\section{Experiment 4}

The results of Experiments 1 and 2 suggested strong constraints of rules on trial-to-trial priming effects. Thus, it is not too surprising that in Experiment 3, we found similar constraints in case of longer term, cumulative learning effects. However, such similarity between the short-term and the long-term also raises an interesting issue. In Experiments 1 and 2, we found that short-term priming benefits were completely constrained by abstract rules. If the same were to happen for longer term effects, this would imply zero transfer of action-specific practice effects across rules. From the viewpoint of extant models of long-term memory (LTM) retrieval of such a finding would be surprising. Typically, speed and probability of retrieving a specific memory trace is assumed to be a monotonic function of the similarity between cue and memory trace (e.g., Hintzman, 1986; Nosofsky \& Palmeri, 1997). However, the possible role of abstract action rules in determining actionrelevant knowledge has not been examined. Thus, it is possible that rule-action conjunctions prove to be an exception to the general rule of similarity-based retrieval. On the one hand, if cumulative priming effects actually do show the same nonmonotonic similarity function as the immediate priming effects, then this would point to an important boundary condition regarding the functional properties of LTM retrieval. On the other hand, if cumulative priming effects of rule-action conjunctions show the traditional monotonic similarity function, this would point to an 
important difference in the representational basis of immediate priming versus long-term priming effects.

In Experiment 4, we adapted our experimental procedure so that we could assess not only learning of action-rule conjunctions but also generalization of such learning across rule boundaries (see Figure 4). For each of the four rules used, one location occurred with higher frequency than any of the other three locations (i.e., the conjunction probes). Each high-frequency conjunction had a lowfrequency twin that served as the generalization probe and that differed only in the rule but not in the stimulus and the response (e.g., upper left stimulus position and upper right response position for both the horizontal and the clockwise rule). The two remaining stimulus locations for each rule were neither high-frequency conjunctions nor twins of high-frequency conjunctions from other rules. These constellations served as controls for the generalization probes. Better performance in conjunction probes than in generalization probes would provide a replication of the conjunction learning result obtained in Experiment 3. More important, better performance in generalization probes than on control trials would suggest generalization of low-level action knowledge across rule boundaries.

Experiment 3 revealed some degree of awareness of conjunction frequencies. However, awareness did not seem to be critical for the emergence of RT benefits associated with high-frequency conjunctions. In Experiment 4, we wanted to further assess the role of awareness. We ran this experiment in two versions, one with a secondary tone-counting task that is known to reduce awareness of implicit regularities (e.g., Mayr, 1996) and one without a secondary task. Our goal was to establish a larger variation in awareness than the one in Experiment 3 (i.e., when combining across the two experiments) and to block awareness when the secondary task was present.

\section{Method}

Participants. In exchange for course credits or $\$ 7,32$ University of Oregon students participated in a single-session experiment.

Task and stimuli. We used the same tasks, stimuli, and cues that we used in Experiments 1-3. We selected rules randomly, with the constraint of no immediate rule repetitions. We selected stimuli conditional on the current rule. For each rule, one of the four possible stimulus locations

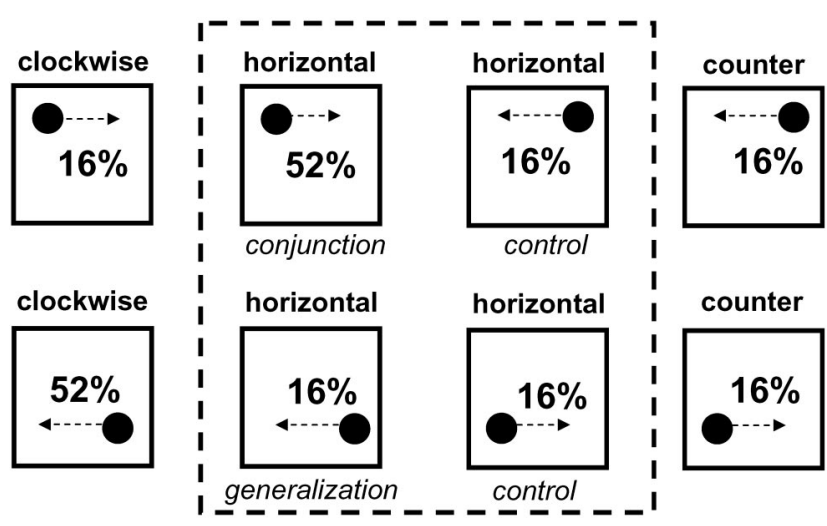

Figure 4. Design of Experiment 4. Stimuli and corresponding frequencies for one particular rule (horizontal) are shown along with those constellations from two other rules to which constellations of the horizontal rule serve as generalization or control constellations. occurred with high probability ( $p=.50$; i.e., the conjunction probes), whereas the other three occurred with lower probability (each $p=.125)$. In addition, for each rule, there was a low-frequency conjunction that entailed the same S-R coupling as a high-frequency conjunction under another rule (i.e., the generalization probes). Finally, there were two control locations for each rule that occurred with low frequency and did not serve as twins of high-frequency conjunctions. The assignment of rules and locations to different conditions was counterbalanced across participants. RCI was 800 $\mathrm{ms}$, and the CSI was $200 \mathrm{~ms}$.

In addition to the primary task, half of the participants also executed a secondary tone-counting task. A 100-ms high or low tone sounded after each response. The task was to count the high and ignore the low tones. After each block, participants were asked through an onscreen dialog box to enter the number of tones counted. In the no-counting condition, participants also heard the tones but were asked to ignore them.

Procedure. Participants worked through two 50-trial practice blocks with equal frequencies across conjunctions and then through eighteen 50-trial experimental blocks followed by the same frequency-judgment task as in Experiment 3.

\section{Results and Discussion}

We excluded error trials and trials after an error, as well as all RTs larger than $4,000 \mathrm{~ms}$ (i.e., $0.9 \%$ of all trials). To analyze frequency effects on performance, we combined the 18 experimental blocks into six 3-block segments.

General effects. Figure 5 shows the critical data. As can be seen, RTs to conjunction probes were fastest, followed by RTs to generalization probes, which in turn were faster than control probes. We confirmed this impression in two separate ANOVAs. Both tested the factors secondary task and a linear practice contrast (i.e., across the six 3-block segments); however, one tested the conjunction versus generalization probes, and the other tested the generalization versus control probes. Each of the two critical frequency contrasts was highly reliable, conjunction versus generalization probes: $F(1,30)=34.91, M S E=7,236.2$; generalization versus control probes: $F(1,30)=16.5, M S E=10,864.7$. Also, the practice effect was highly reliable (both $F_{\mathrm{s}}>30$ ), whereas there were no reliable secondary-task effects (both $F$ s $<$ 1.2) and no other reliable interactions. The two frequency contrasts were also reliable for errors, conjunction versus generalization probes: $F(1,30)=10.74, M S E=1,203.94$; generalization versus control probes: $F(1,30)=4.60, M S E=11,148.68$. Error rates for the control, generalization, and conjunction probes were $5.9 \%$ $(S D=8.1), 3.6 \%(S D=3.7)$, and $2.5 \%(S D=3.5)$, respectively. These results replicate the findings from Experiment 3 showing learning of rule-action conjunctions. More important, they also demonstrate that this type of learning generalizes to some degree across rule boundaries.

Acquisition functions. The absence of interactions between the practice and the two frequency contrasts might indicate that the effect of conjunction frequency is not a skill-acquisition effect but, rather, a general effect that is relevant throughout. For example, on average, there is a shorter lag between a specific conjunction and its last occurrence (or the occurrence of its different-rule twin) for high-frequency conjunctions than there is for low-frequency conjunctions. Thus, the effects we observed possibly reflect short-term priming that spans across trials, but not skill acquisition. However, the manner in which data were presented so far may be misleading, because it ignores that by the power law of practice (e.g., Logan, 1990), most of the learning benefits occur early in practice. 
A

B

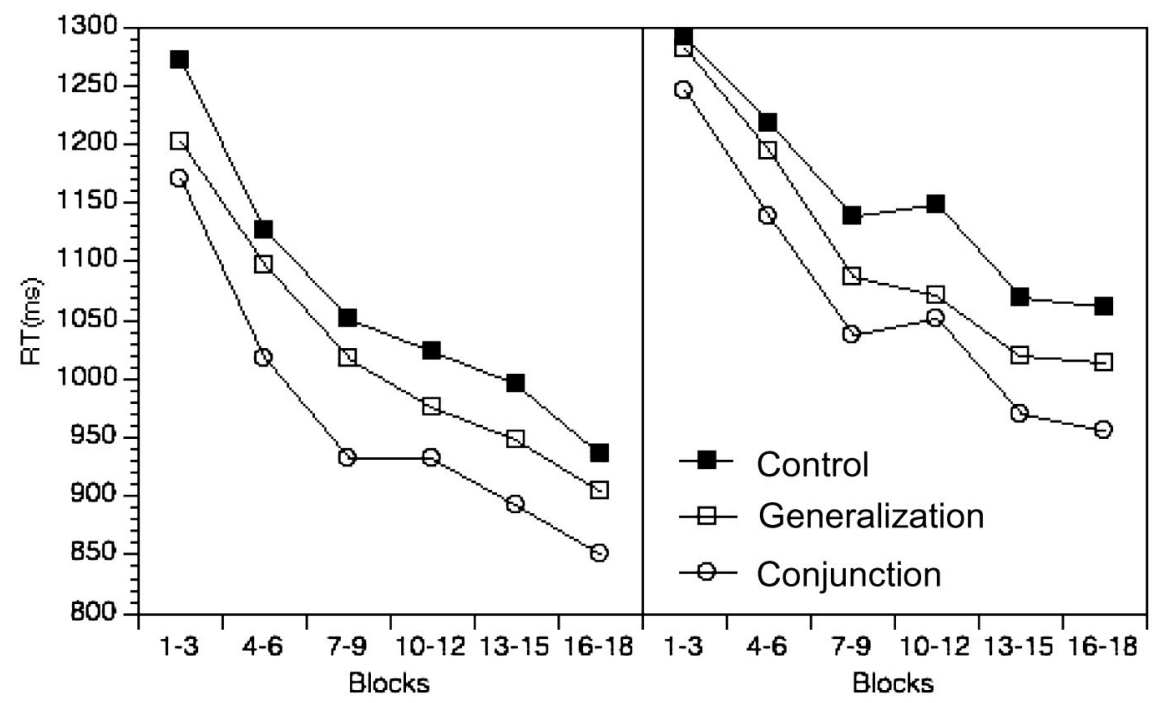

Figure 5. Mean response times (RTs) for the single-task group (A) and the dual-task group (B) of Experiment 4 as a function of condition (conjunction, generalization, and control) and practice (i.e., block segments).

To examine this issue, we plotted in Figure 6 RTs as a function of the number of different types of conjunction repetitions in $\log$-log space. We show only data up to the number of repetitions for which sufficient data were available across participants and conditions $(<35$ for control and generalization and $<90$ for conjunction probes). Of course, for high-frequency conjunction probes, there was a larger number of repetitions per time unit than for control and generalization probes. Regression lines represent power-function fits for each of the three conditions (which are linear in log-log space) computed only for those numbers of repetitions for which data from all conditions were available. As

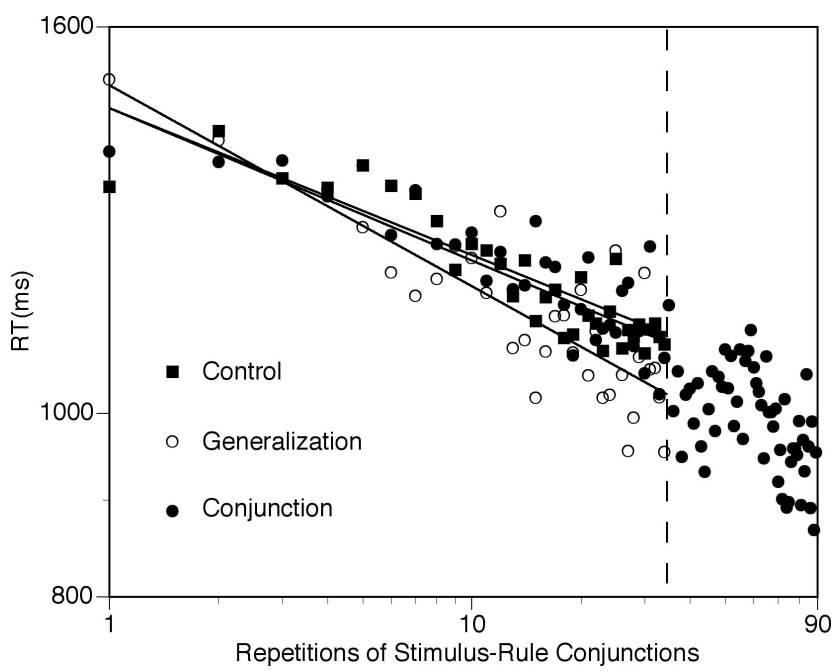

Figure 6. Mean response times (RTs) as a function of condition (conjunction, generalization, and control) as well as number of repetitions of specific stimulus-response rule constellations along with best-fitting linear functions (which in log-log space corresponds to power function fits for untransformed data). can be seen, regression lines were almost identical for conjunction probes and for control probes. This is to be expected if the main determinant of learning is the number of repetitions and not the average lag between repetitions (which, of course, is smaller for conjunction probes than for control probes). Generalization probes showed a steeper slope than the other two probes. This, in turn, is to be expected if generalization probes do actually profit from the cumulative experience with their high-frequency conjunction twins. Finally, the intercept of the three curves with the $y$-axis, the starting point of learning, is remarkably similar. This suggests that the frequency effects we observe actually are driven by cumulative experience rather than by an experience-independent priming effect.

For a statistical test of these observations, we linearly regressed $\log$-RTs on the log of the number of repetitions for each subject and condition. We submitted the resulting individual intercept and slope coefficients to separate $t$ tests for the two different frequency contrasts. Consistent with the group-level pattern, there was no reliable difference between intercept parameters (both $t \mathrm{~s}<0.3$ ); however, the generalization-probe slope was reliably steeper than the control slope, $t(31)=2.38$, whereas the conjunction slope was nearly identical to the control slope, $t(31)=0.18, p>.70$.

Finally, it should be pointed out that when we take into regard the entire learning curve for high-frequency conjunctions (up to 89 repetitions), the resulting log-log regression is actually steeper than the slope for the control condition $t(31)=3.98$. Also, the resulting intercept is reliably larger than that of the control condition, $t(31)=2.65$. This inflated intercept value suggests that learning of high-frequency conjunctions over the entire range of repetitions is not perfectly captured by the power function. There seems to be a speed-up late in practice beyond what the power function would predict and which pulls the function upward for the initial part of the learning curve. We do not have a good explanation for this pattern, other than referring to the possibility of distinct underlying learning processes during different stages of acquisition (e.g., 
Delaney, Reder, Staszewski, \& Ritter, 1998). However, this issue does not affect the central conclusion we would like to draw from the analysis of acquisition functions: Effects of rule-action conjunction frequency are largely due to cumulative experience and not a result of steady-state priming.

Correlational evidence. One possible interpretation of the fact that low-frequency actions benefit from identical high-frequency rule-action conjunctions that occur under different rules is that both conjunction learning and transfer across rule boundaries reflect the same knowledge representation. However, it is also possible that separate representations, one rule-dependent, and the other rule-independent, are acquired. One way to look at this issue is to examine the interrelation between conjunction and generalization learning scores. If this relationship is strong, a common representation seems likely. We correlated RTs and error scores for conjunction probes and generalization probes while partialing out both RTs and error scores for control probes and a variable indicating experimental condition (i.e., secondary task). Thus, only interindividual variability can contribute to this correlation that is specific to the generalization and the conjunction probe condition beyond the interindividual variability captured by the control condition. The resulting correlation was $r=.65, p<.001$, for RTs and $r=.67, p<.001$, for errors. These results are consistent with the assumption that both learning of integrated rule-action conjunctions and the observed rule-independent learning of simple actions reflect a common representation.

It is useful to look back at the corresponding correlations for the immediate priming effects from Experiments 1 and 2. We regressed RTs for S-R and rule repetitions on RTs for rule-only repetitions and $\mathrm{RT}$ s for $\mathrm{S}-\mathrm{R}$-only repetitions on $\mathrm{S}$-only repetitions. The residuals of these two analyses capture the interindividual variability associated with either complete repetitions or S-R-only repetitions, while controlling for the variability contained in adequate control conditions. ${ }^{2}$ The correlation between these two residual scores after we partialed out a variable dummy-coding for experiment was $r=-.18, p>.3$.

Correlations between conjunction and generalization-probe learning scores were highly reliable, suggesting a common representation. In contrast, correlations between S-R-rule repetition priming scores versus $\mathrm{S}-\mathrm{R}$-only priming scores were basically nonexistent, suggesting representations that are separated by rule boundaries.

Awareness of frequencies. During the final frequencyawareness assessment, participants had to indicate for each rulestimulus conjunction whether it occurred with low or high frequency. Non-secondary-task participants gave a "high-frequency" answer to $57.8 \%(S D=19.2)$ of the control conjunctions, $64.6 \%$ $(S D=20.3)$ of the generalization probes, and $73.4 \%(S D=24.9)$ of the high-frequency conjunctions. The corresponding values for the secondary-task group were $60.9 \%(S D=18.7), 57.8 \%(S D=$ $17.6)$, and $65.6 \%(S D=22.1)$. Numerically, it seems that in the absence of the secondary task, some awareness of the conjunction frequencies was achieved, whereas under a secondary task, the corresponding effects were much lower. However, statistically, there was little evidence for frequency awareness. The difference between generalization-probe scores and control-probe scores was reliable for neither group, $t(15)<0.6$. The difference between high-frequency conjunction and control probes was not reliable for the secondary-task group, $t(15)=0.9$, but approached reliability for the non-secondary-task group, $t(15)=1.89, p<.08$. In order to examine the possible role of explicit awareness for the observed RT effects, we correlated each of the two explicit scores with the corresponding RT scores across both secondary-task conditions (in each case, generalization-probe minus control and high-frequency conjunction minus control). For the generalization condition, the correlation was $r=.05$, and for the high-frequency condition, it was $r=-.04$. Thus, there is no positive evidence that awareness influenced RT learning.

These results may have to be taken with a grain of salt. It is likely that reliability of awareness scores is low, and we cannot rule out the possibility that influences of awareness might be detected with more reliable measures. At the same time, the current results replicate those of Experiment 3 with regard to the absence of any trace of a relationship between reported assessments of conjunction frequencies and RT frequency effects.

\section{Abstract Rules Versus Cue-Stimulus Compounds?}

A critical assumption underlying all of our experiments is that participants solve the spatial-rules task by using the abstract rules to perform the adequate transformations. Only if that was the case can we take the evidence reported in Experiments 1-4 to suggest integration between rules and specific actions. However, Logan and Bundesen (2003) recently claimed that in the cued taskswitching paradigm, participants do not actually select between the different tasks on a trial-by-trial basis (see also the Discussion of Experiments 1 and 2 section). Rather, these authors suggested that participants use the cue-stimulus compound to determine the correct response in a purely associative manner. If this is what participants did in our experiments, our results should be interpreted in terms of compounds between cues, stimuli, and responses but not necessarily in terms of integration between codes representing abstract task sets on one hand and stimuli-responses on the other.

Whether participants use abstract rules is not always easy to determine (but see Duncan, 1978). However, in our paradigm, there is an empirical phenomenon that should be helpful in this regard, namely the effect of rule difficulty. The horizontal and vertical rules are each associated with two sets of symmetric transformations (i.e., move left vs. move right and move up vs. move down), whereas the clockwise and the counterclockwise rule are each associated with four possible translation directions (conditional on the four possible circle positions), which do not form symmetric pairs. Therefore, the latter two rules should be more difficult to apply than the former two rules, that is, of course, if participants actually made use of these rules. If, however, response selection is a purely associative process, in which the cue and the stimulus together serve as a compound stimulus associated with a particular response, no such difficulty effect is to be expected.

Across all four experiments, we found difficulty effects between the two sets of rules of around $100 \mathrm{~ms}$ or more (Experiments 1-4: $91 \mathrm{~ms}, 115 \mathrm{~ms}, 113 \mathrm{~ms}$, and $168 \mathrm{~ms}$, respectively; all $t$ values > 3.0). This effect was present in substantial magnitude in the final third of each experiment $(115 \mathrm{~ms}, 69 \mathrm{~ms}, 66 \mathrm{~ms}, 135 \mathrm{~ms}$, respec-

\footnotetext{
${ }^{2}$ It is somewhat ambiguous what exactly the best control condition for the S-R-only repetition RTs is. However, whether one uses the stimulusonly repetitions (as reported in the text), any of the other possible conditions (i.e., response-only repetitions or complete nonrepetitions), or the average of these three conditions does not affect the pattern of results.
} 
tively; all $t$ values $>3.0$ ). Thus, rule use seems to dominate performance throughout and not just in the first few blocks where direct associations may still have to be established. In general, these difficulty effects speak against the idea that participants perform the task via simple stimulus-response associations (which, of course, are identical across easy and difficult rules).

Finally, note that in the Discussion of Experiments 1 and 2 section, we refer to new data that showed the characteristic pattern of set-dependent priming even in a situation in which no external cues were present. Thus, all in all, we feel that the interpretation of our results in terms of evidence for integration between abstract rules and lower level stimulus-response codes is justified. As one potential qualification, we note that in Experiments 3 and 4 (on skill acquisition), we used an external cuing paradigm. Thus, in principal, these experiments are open to the critique that only the sensory aspects of cues and not the codes that represent abstract task sets are responsible for the learning effects. However, the fact that immediate priming effects did not depend on the presence of external task cues and that rule difficulty affected performance throughout suggests that participants were using abstract rules. Therefore, it is a plausible, though not yet sufficiently tested, assumption that these rule-level settings were also involved in the learning process.

\section{General Discussion}

With this series of experiments, we wanted to determine how abstract rules interact with representations of specific actions. To be specific, we examined the degree to which abstract rules constrain trial-to-trial stimulus-response priming effects and cumulative practice effects.

\section{Rule-Action Conjunctions and Trial-to-Trial Priming}

Our starting point was the observation obtained in taskswitching studies that repetition-priming benefits turn into costs in case of a change in task. On one hand, this finding is consistent with the idea that specific actions and abstract rules form an integrated representation that serves as the basis of the repetitionpriming effect (e.g., Meiran, 2000; Schuch \& Koch, 2004). On the other hand, such an interaction could arise from the fact that a change in abstract rules or task set usually coincides with a change in relevant stimulus attributes. As a consequence, stimulusresponse associations established on one trial need to be uncoupled to allow the same response to be associated with a different stimulus attribute on the next trial (e.g., Hommel, 2004). In this case, abstract rules would modulate repetition priming indirectly, namely via the effects of attentional settings that coincide with these rules, but not by becoming part of an integrated actionrelevant representation.

Our paradigm allowed us to establish trial-to-trial transitions in which abstract rules changed, whereas the entire stimulusresponse ensemble repeated. We found that even in this case, a repetition cost instead of a benefit was obtained. This result suggests that abstract rules are combined with stimulus and response rules into a common representation that serves as the basis for immediate repetition-priming effects. Thus, a strictly hierarchical mode of control in which selection on the rule level would only set the constraints for the response-selection level can be rejected. If such a model was correct, then repetition-priming effects should have been solely based on the trial-to-trial similarity of stimulus and response parameters.

It is interesting to note that there are several findings in the task-switching literature that seem to support a strict separation between set-level selection and response selection (e.g., Kray \& Lindenberger, 2000; Gopher et al., 2000). Most notably, switch costs are usually unaffected by manipulations of responseselection difficulty. However, such dissociation evidence reflects what is going on during selection. In contrast, the Task Switching $\times$ Response Priming interaction reflects aftereffects of selection. This leaves room for the following scenario. A distinct set of selection processes may be relevant for determining the to-beattended stimulus attributes (Corbetta, Miezin, Dobmeyer, Shulman, \& Petersen, 1991; Weidner, Pollmann, Mueller, \& von Cramon, 2002), response codes (Rushworth, Hadland, Paus, \& Sipila, 2002), and abstract rules that set the constraints for selection of lower level codes (e.g., Christoff \& Gabrieli, 2000). Once the ensemble of codes for a particular action is determined, it becomes integrated. It is possible that this integration is a precondition for execution of action. However, it is also possible that integration is just an aftereffect of codes being simultaneously active.

\section{Rule-Action Conjunctions and Skill Acquisition}

In Experiments 3 and 4, we examined to what degree cross-level integration is also relevant for cumulative priming (skill acquisition) effects. Past work had already demonstrated that cumulative priming effects depend on the way a stimulus is interpreted (Logan, 1990). However, again it is not clear whether this evidence suggests direct involvement of set-level codes in the learning process or whether task sets determine only which stimulus aspects participate in learning but do not themselves become part of the knowledge base. We found that high-frequency conjunctions between abstract rules and specific S-R couplings were performed faster than identical S-R couplings that occurred under different rules. This result strongly suggests that codes representing rules or task sets can become part of the skill-relevant knowledge base. It is interesting to note that Waszak, Hommel, and Allport (2003) reported a related result from a very different paradigm. Using a picture-word interference task, they showed that switch costs were particularly large when participants had to name the word of a picture-word combination in which the picture had been used earlier in the context of a picture-naming task. Congruent with the current finding, this result suggests that a task set (e.g., picture naming) can become associated with a stimulus to which it has been applied.

An additional interesting aspect is the role of awareness during learning of rule-S-R conjunctions. Though not definitive, our results suggest that highly contextualized, conjunctive memories can arise in the absence of conscious encoding or recollection. Medial temporal brain areas are assumed to be critical for the coding of conjunctions (Sutherland \& Rudy, 1989) but are often also associated with conscious, declarative memories (e.g., Squire \& Zola-Morgan, 1991). Thus, this paradigm may be useful in brain imaging or patient work looking at potential, medial-temporal contributions to the formation of conjunctive but nondeclarative memories (e.g., Chun \& Phelps, 1999). 


\section{Immediate Priming Versus Skill Acquisition}

If abstract rules have the same complete effect on skill acquisition as they have on trial-to-trial priming, then learning would be extremely specific, allowing no generalization across rule boundaries. Moreover, this would contradict the typical way in which retrieval of information from LTM is usually conceptualized, namely as a monotonic function of similarity between retrieval cue and memory trace (e.g., Nosofsky \& Palmeri, 1997). The results we report in Experiment 4 were in fact consistent with similaritybased retrieval for the case of rule-specific skill acquisition effects. Responses were fastest for high-frequency repetitions of rules, stimuli, and responses. However, some speed-up was observed even when the S-R coupling was identical to that used in a high-frequency conjunction but the rule was not. This result is good news when it comes to model parsimony. The standard model of LTM retrieval does not have to be modified to handle abstract rules (e.g., Logan, 1988). From the standpoint of memory retrieval, rules are just another feature that determines similarity between a cue and memory traces.

An open question is in what manner abstract rules participate in instances. One possibility is that either abstract rules or the stimuli by which they are indicated (i.e., the task cues) literally serve as nothing more than a feature that determines similarity between a retrieval cue and traces in memory (e.g., Logan and Bundesen 2003). To be specific, because in Experiments 3 and 4 explicit cues were used for each rule, our results on cumulative learning and generalization effects could be explained in this manner. A second, and not mutually exclusive, possibility is that memory instances are not just arrays of stimulus values but, rather, capture the particular constellation of selection processes that have been applied to a certain situation. In this case, retrieval of the instance triggers the reenactment of the original constellation of selection processes, including the rule-based transformations. Thus, here the actual rule would be part of the representation. A definitive answer to this important question about the relationship between rules and associative processes will require further empirical work.

Although our acquisition-related results are generally compatible with extant models of memory retrieval, they do complicate the issue for theories of priming because they suggest that processing benefits from cumulative experiences and processing benefits from the immediate past are governed by different principles. In this context, it should be noted that all-or-none priming patterns have been obtained in a number of different situations (e.g., Campbell \& Proctor, 1993; Mayr, Awh, \& Laurey, 2003; Pashler \& Baylis, 1991; Quinlan, 1999). A frequent account of such patterns is that participants use something akin to a global similarity match between the memory of the most recent selection instance and the current selection instance to determine whether the most recent action can be simply reapplied (i.e., a memory shortcut) or whether the current memory content needs to be abandoned altogether and a new selection process initiated. However, this account offers little more than a redescription of the observed data pattern. The question left unanswered is why information about the most recent selection instance in working memory can be used in an all-ornone manner only. The integration view offers a possible explanation. According to this view, codes that are simultaneously active become integrated into a common representation, sometimes referred to as an event file (Hommel, 2004). This is also in line with broader perspectives regarding the idea that conscious selection for action goes along with the establishment of integrated and internally coherent representations within a global workspace (Dehaene, Kerszberg, \& Changeux, 1999). The very definition of an integrated, coherent representation entails that one cannot simply change one part without affecting the rest. A code of a specific stimulus or response that is part of such representation is not accessible for flexible use outside of the integrated representation-thus the cost in case of a repetition after a task switch (Hommel, 2004).

A somewhat different account of the Switch $\times$ Repetition interaction was suggested by Kleinsorge and colleagues (e.g., Kleinsorge \& Heuer, 1999). They propose that the cost-benefit repetition pattern reflects the way hierarchical control operates. Supposedly, the control architecture requires that for changes on a hierarchically higher level (i.e., the rule level), all lower level parameters need to be changed as well. In contrast, lower level parameters can change without requiring higher level changes. Response-repetition costs in case of a task switch reflect the need to reset inadvertently changed lower level aspects. A problem with this account is that it provides no explanation for why changes of settings generalize downstream. At the same time, the model does account for why changes on the task-set level seem to have more pervasive effects than changes on lower levels. For example, in our Experiments 1 and 2, rule repetitions compared with rule changes always produced benefits, whereas response-stimulus repetitions produced costs or benefits depending on whether the rule changed. According to the Kleinsorge model, this is the case because changes generalize downstream but not upstream. ${ }^{3}$

It is possible that one can combine the Kleinsorge model and the integration account by assuming that integrated representations are hierarchically organized rather than just an unstructured ensemble of codes. Rules could provide the structure for an integrated representation, including open slots for which specific stimulusresponses serve as arguments (e.g., Halford, Bain, Maybery, \& Andrews, 1998). Thus, a rule change abolishes the structure of the integrated representation, whereas a stimulus-response change requires only the exchange of slot values. Because of integration, specific parameters become associated with the rules and thus cannot be easily reused after a change in rule. Recent computational models explicitly conceptualize rules as hybrid entities, which provide structure to a set of variables but which also possess the stickiness of an associative learning system (Hummel \& Holyoak, 2003).

There are many open issues with this general account. An important one concerns the time course of integration-based priming versus the similarity-based, long-term effects. On the basis of the idea that integration happens when codes are coactive in working memory, we might suspect that integrationbased priming occurs as long as the integrated representation is

\footnotetext{
${ }^{3}$ It should be noted that it is possible that the hierarchical effects described by Kleinsorge and Heuer (1999) have less to do with the hierarchical control structure but more to do with the temporal order in which selection processes proceed. In typical task-switching experiments, participants first change the rule and then select the lower level parameters. It is possible that the usual effects of set-selection changes on lower level changes were reversed if participants received stimulus-specific information prior to rule-specific information.
} 
present in working memory. The finding in Experiment 2 of a reduction of the cost-benefit pattern as a function of intertrial interval is consistent with this idea. Future work should determine more precisely what the boundary conditions are for integration-based, short-term priming versus similarity-based, long-term priming.

\section{Conclusion}

We show here that the selection of simple actions is intricately linked to the abstract rule by which it is governed. This general result is inconsistent with the view of a strictly hierarchical mode of control. In addition, the linkage between high-level selection and low-level selection manifests itself in somewhat different ways in the short term (i.e., benefits for only complete repetitions of rule-S-R conjunctions) and the long term (i.e., benefits for complete rule-S-R conjunctions with generalization of $\mathrm{S}-\mathrm{R}$ benefits across rule boundaries). This difference may reflect a fundamental dissociation between the way in which we can make use of information that is currently active, or was very recently active, in working memory (all or none) and the way in which we can make use of information from long-term memory (in a graded, similarity-based manner).

\section{References}

Allport, D. A., Styles, E. A., \& Hsieh, S. (1994). Switching intentional set: Exploring the dynamic control of tasks. In C. Umiltà \& M. Moscovitch (Eds.), Attention and performance XV (pp. 421-452). Hillsdale, NJ: Erlbaum.

Campbell, K. C., \& Proctor, R. W. (1993). Repetition effects with categorizable stimulus and response sets. Journal of Experimental Psychology: Learning, Memory, and Cognition, 19, 1345-1362.

Christoff, K., \& Gabrieli, J. D. E. (2000). The frontopolar cortex and human cognition: Evidence for a rostrocaudal hierarchical organization within the human prefrontal cortex. Psychobiology, 28, 168-186.

Chun, M. M., \& Phelps, E. (1999). Memory deficits for implicit contextual information in amnesic subjects with hippocampal damage. Nature Neuroscience, 2, 844-847.

Corbetta, M., Miezin, F. M., Dobmeyer, S., Shulman, G. L., \& Petersen, S. E. (1991). Selective and divided attention during visual discrimination of shape, color, and speed: Functional anatomy by positron emission tomography. Journal of Neuroscience, 11, 2383-2404.

Dehaene, S., Kerszberg, M., \& Changeux, J.-P. (1999). A neuronal model of a global workspace in effortful cognitive tasks. Proceedings of the National Academy of Sciences, USA, 95, 14529-14534.

Delaney, P. F., Reder, L. P., Staszewski, J. J., \& Ritter, F. E. (1998). The strategy-specific nature of improvement: The power law applies by strategy within task. Psychological Science, 9, 1-7.

Duncan, J. (1978). Response selection in spatial choice reaction: Further evidence against associative models. Quarterly Journal of Experimental Psychology A, 30, 429-440.

Gopher, D., Armony, L., \& Greenshpan, Y. (2000). Switching tasks and attention policies. Journal of Experimental Psychology: General, 129, $308-339$.

Halford, G. S., Bain, J. D., Maybery, M. T., \& Andrews, G. (1998). Induction of relational schemas: Common processes in reasoning and complex learning. Cognitive Psychology, 35, 201-245.

Hintzman, D. (1986). "Schema abstraction" in a multiple-trace memory model. Psychological Review, 93, 411-428.

Hommel, B. (2004). Event files: Feature binding in and across perception and action. Trends in Cognitive Sciences, 8, 494-500.

Hummel, J. E., \& Holyoak, K. J. (2003). A symbolic-connectionist theory of relational inference and generalization. Psychological Review, 110, $220-264$.
Kleinsorge, T., \& Heuer, H. (1999). Hierarchical switching in a multi-dimensional task space. Psychological Research, 62, 300312.

Koechlin, E., Ody, C., \& Kounelher, F. (2003, November 14). The architecture of cognitive control in the human prefrontal cortex. Science, 302, $1181-1185$.

Kray, J., \& Lindenberger, U. (2000). Adult age differences in task switching. Psychology and Aging, 15, 126-147.

Logan, G. D. (1988). Toward an instance theory of automatization. Psychological Review, 95, 492-527.

Logan, G. D. (1990). Repetition priming and automaticity: Common underlying mechanisms? Cognitive Psychology, 22, 1-35.

Logan, G. D., \& Bundesen, C. (2003). Clever homunculus: Is there an endogenous act of control in the explicit task-cuing procedure? Journal of Experimental Psychology: Human Perception and Performance, 29, 575-599.

Marczinski, C. A., Milliken, B., \& Nelson, S. (2003). Aging and repetition effects: Separate specific and nonspecific influences. Psychology and Aging, 18, 780-790.

Mayr, U. (1996). Spatial attention and implicit sequence learning: Evidence for independent learning of spatial and nonspatial sequences. Journal of Experimental Psychology: Learning, Memory, and Cognition, 19, 1297-1320.

Mayr, U. (2002). Inhibition of action rules. Psychonomic Bulletin \& Review, 9, 93-99.

Mayr, U., Awh, E., \& Laurey, P. (2003). Does conflict adaptation require executive control? Nature Neuroscience, 6, 450-452.

Mayr, U., \& Keele, S. (2000). Changing internal constraints on action: The role of backward inhibition. Journal of Experimental Psychology: General, 129, 4-26.

Mayr, U., \& Kliegl, R. (2000). Task-set switching and long-term memory retrieval. Journal of Experimental Psychology: Learning, Memory, and Cognition, 26, 1124-1140.

Mayr, U., \& Kliegl, R. (2003). Differential effects of cue changes and task changes on task-set selection costs. Journal of Experimental Psychology: Learning, Memory, and Cognition, 29, 362-372.

Meiran, N. (1996). Reconfiguration of processing mode prior to task performance. Journal of Experimental Psychology: Learning, Memory, and Cognition, 22, 1423-1442.

Meiran, N. (2000). Reconfiguration of stimulus task-sets and response task-sets during task-switching. In S. Monsell and J. Driver (Eds.), Control of cognitive processes: Attention and performance XVIII (pp. 377-400). Cambridge, MA: MIT Press.

Norman, D. A., \& Shallice, T. (1986). Attention to action: Willed and automatic control of behavior. In R. J. Davidson, G. E. Schwartz, \& D. Shapiro (Eds.), Consciousness and self-regulation, Vol. 4 (pp. 1-18). New York: Plenum.

Nosofsky, R. M., \& Palmeri, T. J. (1997). An exemplar-based random walk model of speeded classification. Psychological Review, 104, 266-300.

Pashler, H., \& Baylis, G. (1991). Procedural learning: 2. Intertrial repetition effects in speeded-choice tasks. Journal of Experimental Psychology: Learning, Memory, and Cognition, 17, 33-38.

Quinlan, P. T. (1999). Sequential effects in auditory choice reaction time tasks. Psychonomic Bulletin \& Review, 6, 297-303.

Rogers, R. D., \& Monsell, S. (1995). The cost of a predictable switch between simple cognitive tasks. Journal of Experimental Psychology: General, 124, 207-231.

Rubinstein, J., Meyer, E. D., \& Evans, J. E. (2001). Executive control of cognitive processes in task switching. Journal of Experimental Psychology: Human Perception and Performance, 27, 763-797.

Rushworth, M. F. S., Hadland, L. A., Paus, T., \& Sipila, P. K. (2002). Role of the human frontal cortex in task switching: A combined fMRI and TMS study. Journal of Neurophysiology, 87, 2577-2592. 
Schuch, S., \& Koch, I. (2004). The costs of changing the representation of action: Response repetition and response-response compatibility in dual tasks. Journal of Experimental Psychology: Human Perception and Performance, 29, 92-105.

Squire, L. R., \& Zola-Morgan, S. (1991). The medial temporal lobe memory system. Science, 253, 380-1386.

Sutherland, R. J., \& Rudy, J. W. (1989). Configural association theory: The role of the hippocampus formation in learning, memory, and amnesia. Psychobiology, 17, 129-144.

Waszak, F., Hommel, B., \& Allport, A. (2003). Task-switching and long- term priming: Role of episodic stimulus-task bindings in task-shift costs Cognitive Psychology, 46, 361-413.

Weidner, R., Pollmann, S., Mueller, H. J., \& von Cramon, D. Y. (2002). Top-down controlled visual dimension weighting: An event-related fMRI study. Cerebral Cortex, 12, 318-328.

Received March 12, 2004

Revision received September 10, 2004

Accepted September 24, 2004

\section{Thomas O. Nelson (1943-2005)}

We regret to inform our readers that Thomas $\mathrm{O}$. Nelson, $\mathrm{PhD}$, editor of Journal of Experimental Psychology: Learning, Memory, and Cognition, died on January 14, 2005, of complications from an aortic aneurysm. Dr. Nelson had a distinguished publication history and focused his research on human memory, metacognition, and computer-assisted instruction for learning foreign-language vocabulary. In 1995, Dr. Nelson joined the faculty in the psychology department of the University of Maryland, College Park, and led the Cognitive Program. Dr. Nelson had been an APA member since 1988 and Fellow of Division 3 (Experimental Psychology) since 1994. A devoted father of two, an avid outdoorsman, and a man of eclectic tastes, Dr. Nelson will be greatly missed. 